\title{
Single Center Experience on Screening Oncology Patients for Covid-19 Before Anti-Cancer Treatment
}

\author{
Esat NAMAL ${ }^{1}$, Nur DINC ${ }^{1}$, Sezer SAGLAM ${ }^{1}$, Ali Vefa OZTURK $^{2}$, Safiye Koculu DEMIR ${ }^{3}$, \\ Asiye YIR ${ }^{3}$, Esin CEVIK ${ }^{3}$, Soheyl SABET ${ }^{4}$, Bedriye Koyuncu SOKMEN ${ }^{4}$, Nagihan Inan GURCAN ${ }^{4}$, \\ Yaman TOKAT ${ }^{5}$, Cavlan CIFTCI ${ }^{6}$
}

\author{
${ }^{1}$ Demiroglu Bilim University, Faculty of Medicine, Department of Medical Oncology \\ ${ }^{2}$ Demiroglu Bilim University, Faculty of Medicine, Department of Pulmonary Disease \\ ${ }^{3}$ Demiroglu Bilim University, Faculty of Medicine, Department of Infectious Disease \\ ${ }^{4}$ Demiroglu Bilim University, Faculty of Medicine, Department of Radiology \\ ${ }^{5}$ Demiroglu Bilim University, Faculty of Medicine, Department of Liver Transplantation \\ ${ }^{6}$ Demiroglu Bilim University, Faculty of Medicine, Department of Cardiology, Istanbul, TURKEY
}

\begin{abstract}
The most important factor in the transmission of the COVID-19 is asymptomatic carriers. We've tested all oncology patients , that receive anti-cancer therapy, for COVID-19. We aimed to determine the rate of asymptomatic carriers, and analyze the clinical and radiological findings of infected patients. Oncology patients who have indications of receiving anti-cancer treatment in the hospital were tested for COVID-19, two day prior to their treatment even if they were asymptomatic by collecting nasopharyngeal and oropharyngeal swab specimens for RT-PCR for viral RNA detection. Positive patients, underwent inspiratory phase of chest computed tomography examination. Infected patients were given the recommended treatment for COVID-19. PCR test was positive in 28 of 312 patients that we tested, and the positivity rate was $8.9 \%$. Three patients (10.7\%) had symptoms, 25 patients (89.3\%) had no symptoms. Covid-19 testing before anti-cancer treatment may be recommended in order to continue their treatment without any problems and to prevent the risk of transmission due to the high rate of asymptomatics in infected patients.
\end{abstract}

Keywords: COVID-19, Pandemic, Chemotherapy, Oncology

\section{INTRODUCTION}

Coronaviruses $(\mathrm{CoV})$ are RNA viruses that can be pathogenic in humans and animals. The novel $\mathrm{CoV}$ which is called severe acute respiratory syndrome coronavirus 2 (SARS-CoV-2), was identified to transmit from human to human at the end of 2019, and viral pneumonia associated with SARS-CoV-2 started to appear in Wuhan, China. ${ }^{1}$ The World Health Organization (WHO) named this disease as COVID-19, which means $2019 \mathrm{CoV}$ disease $^{2}$, and declared it to be a pandemic in March 2020.
People infected with COVID-19 can have a wide range of clinical manifestations, from asymptomatic disease to follow-up indication in intensive care unit. The most feared outcome is viral pneumonia, followed by acute respiratory distress syndrome (ARDS) and death. ${ }^{3}$ The severe course of the infection is more common in patients with comorbidity and in the elderly. ${ }^{4}$ Person-to-person contamination generally occurs by inhaling droplets in the air during close contact or by touching the mouth, eyes, or nose after touching an infected surface. ${ }^{5}$ 
Although the data on the disease have not matured yet, in an analysis in Italy, 20 percent of COVID-19 deaths in the entire country were in cancer patients. ${ }^{6}$ In another study conducted in China, 18 of 2007 COVID-19 infected patients were cancer patients and the infection was reported to cause serious clinical events more frequently in cancer patients compared to the general populations. ${ }^{7}$

During the COVID-19 pandemic, some arrangements were made in the treatment of cancer patients treated in our center in line with the recommendations of international and national cancer associations. ${ }^{8,9}$ Accordingly, oral treatments were preferred as much as possible, in order to prevent entries of patients to the hospital, and chemotherapy was avoided in patients who were expected to have a low survival benefit from treatment. We changed the nonurgent follow-up visits to telemedicine visits, via video or phone.

Despite the lack of sufficient evidence, important associations such as European Society for Medical Oncology (ESMO) and Infectious Diseases Society of America (IDSA) recommended that patients undergoing active oncological treatment should be tested for COVID-19 before each treatment. ${ }^{10,11}$ Based on these recommendations, all patients who were receiving anti-cancer treatments in our hospital were tested for COVID-19 two day prior to their treatment even if they were asymptomatic, by collecting nasopharyngeal and oropharyngeal swab specimens for reverse-transcription polymerase chain reaction (RT-PCR) for viral RNA detection. The reason for this is to prevent oncology patients, who have to come to the hospital periodically and may be having an asymptomatic infection, from spreading infection and to make the decision to postpone chemotherapy in infected patients. After this screening, we aimed to retrospectively evaluate the clinical and radiological findings of oncology patients determined to be infected with COVID-19 and to contribute to this area with limited data.

\section{PATIENTS AND METHODS}

COVID-19 screening was performed on 312 oncology patients that actively receiving anti-cancer treatments via nasopharyngeal and oropharyngeal swab specimens for COVID-19 RT-PCR.

28 patients, who were positive for SARS-CoV-2, underwent chest computed tomography (CT) examination with SOMATOM Go Now (Siemens Medical Solutions, Erlangen, Germany). The technical parameters were as following: tube voltage, 100-140 kVp; tube current, 100-250 mA; and tube rotation time, $0.8 \mathrm{~s}$, slice thickness $5 \mathrm{~mm}$, with 1 $\mathrm{mm}$ or $1.5 \mathrm{~mm}$ section thickness for axial, coronal and sagittal reconstructions. All images were assessed by two radiologists (SS, NI) who were blinded to the clinical history and laboratory results. The findings classified by the consensus of two radiologists. On visual evaluation, the pattern of the abnormal attenuation as well as the involved lung lobes were recorded. For the quantification, the percentage of involvement lung volume (ILV) occupied by ground glass opacity (GGO) and / or consolidation areas and total lung volume (TLV) were calculated by automatically using commercial software, Myrian ${ }^{\circledR}$ (Intrasense, Montpellier, France). Lung volume (LV) can be measured as lung volume with attenuation from -950-700 HU, the ILV volume can be measured as lung volume with attenuation from -800-500 HU.

Patients who were receiving anti-cancer treatment and positive PCR results were routinely given hydroxychloroquine $2 \times 400 \mathrm{mg}$ tablet on the first day for loading, 2 x $200 \mathrm{mg}$ tablet for maintenance, oseltamivir $2 \times 75 \mathrm{mg}$ tablet, azithromycin $1 \times 500$ $\mathrm{mg}$ tablet first day and for maintenance $1 \times 250 \mathrm{mg}$ tablet for a total of 5 days. Favipravir $2 \times 1600 \mathrm{mg}$ tablet first dose, and for maintenance $2 \times 600 \mathrm{mg}$ tablet were added to the treatment of one patient requiring hospitalization. Hydroxychloroquine was closely monitored for side effects in terms of arrhythmia.

The anti-cancer treatment of all patients who had positive PCR results was postponed for 14 days. At the end of 14 days, patients with two negative PCR tests with an interval of one week were considered as recovered and their anti-cancer treatments continued.

Descriptive statistical data, including mean, standard deviation, median, minimum and maximum were used to describe continuous variables, while 
percentages was used for the percentages of categorical variables. Analyses were performed using MedCalc Statistical Software version 12.7.7.

The institutional review board of this hospital approved our study, and informed consent was waived due to the retrospective study design.

\section{RESULTS}

COVID-19 PCR test was performed on 312 oncology patients, who were actively receiving anti-cancer therapy at Demiroğlu Bilim University Medical Oncology Clinic, two days prior to the anti-cancer treatment. PCR results of 28 patients (8.9\%) were evaluated as positive, and the clinical, radiological, laboratory and demographic characteristics of these patients were analyzed. 14 of the patients were females and 14 were males. The mean age was $57.6 \pm$ 12.5. 7 patients were diagnosed with breast, 6 with colon, 5 with lung, 3 with stomach, 2 with ovarian, 2 with pancreatic cancer, 1 with cholangiocarcinoma, 2 with sarcoma. Eleven patients had distant metastases and were receiving palliative therapy, while 17 patients were receiving adjuvant therapy. 23 patients had cytotoxic chemotherapy and 5 patients had targeted treatment in the preceding 3 weeks. Each patient was questioned for symptoms of fever, cough, dyspnea, muscle pain, headache, changes in taste and smell. Three patients (10.7\%) had symptoms; 2 of whom had dyspnea and cough, and 1 had headache, and 25 patients $(89.3 \%)$ had no symptoms. As the patients under chemotherapy, almost all patients complained of taste disturbance, but this was thought to be due to their oncological treatment. Six patients (21.4\%) had lymphopenia and three with lymphopenia had elevated c reactive protein (crp) and one had leucocytosis. Comorbidities were present in $32 \%$ of the patients including diabetes mellitus in 2 patients, hypertension in 5 patients, and autoimmune diseases in 2 patients (Table 1). In 15 (53.5\%) of 28 patients, the people they lived with at home could not fully comply with social isolation due to compulsory reasons and $12(42.8 \%)$ patients came to the hospital by using public transport. Anti-cancer treatment was interrupted in all patients.

All of the PCR-positive patients received low-dose

\begin{tabular}{|c|c|}
\hline Variables & n (\%) \\
\hline \multicolumn{2}{|l|}{ Sex } \\
\hline Male & 14 \\
\hline Female & 14 \\
\hline Age & $57.6 \pm 12.5$ \\
\hline \multicolumn{2}{|l|}{ Primary site of cancer } \\
\hline Breast cancer & 7 \\
\hline Colorectal cancer & 6 \\
\hline Lung cancer & 5 \\
\hline Gastric cancer & 3 \\
\hline Ovarian cancer & 2 \\
\hline Pancreatic cancer & 2 \\
\hline Cholangiocarcinoma & 1 \\
\hline Soft tissue sarcoma & 2 \\
\hline \multicolumn{2}{|l|}{ Stage of cancer } \\
\hline Metastatic disease & 11 \\
\hline Non metastatic disease & 17 \\
\hline \multicolumn{2}{|l|}{ Anti-cancer treatment } \\
\hline Chemotherapy \pm targeted therapy & 23 \\
\hline Only targeted therapy & 5 \\
\hline \multicolumn{2}{|l|}{ Symptoms } \\
\hline Asymptotic & 25 (89.3\%) \\
\hline Sympyomatic & $3(10.7 \%)$ \\
\hline \multicolumn{2}{|l|}{ Comorbidity } \\
\hline Diabetes & 2 \\
\hline Hypertension & 5 \\
\hline Autoimmune disease & 2 \\
\hline \multicolumn{2}{|l|}{ Laboratory findings } \\
\hline Lymphopenia & $6(21.4 \%)$ \\
\hline Crp increase & 3 \\
\hline Leukocytosis & 1 \\
\hline \multicolumn{2}{|l|}{ Thorax CT findings } \\
\hline Unremarkable chest CT & $13(46.4 \%)$ \\
\hline Viral pneumonia & $15(53.6 \%)$ \\
\hline
\end{tabular}

CT without contrast. Thirteen (46.4\%) patients had no viral pneumonia, while $15(53.6 \%)$ patients showed suggestive findings for COVID-19 (Table 1). Ten cases had multifocal involvement while five cases had single lesion. Mean total lung volumes were $4263.73 \pm 1243.26 \mathrm{~mL}$ and $3470.74 \pm$ 
$1097.81 \mathrm{~mL}$ in the positive and negative groups, respectively. Mean LV were $2396.41 \pm 771.15$ $\mathrm{mL}$ and $1867.17 \pm 535.41 \mathrm{~mL}$, for the right and left lung respectively in all cases. The mean of involved Lung Volume (ILV) percentage in patients with suggestive CT findings was $8.9 \% \pm 7.8$.

One patient require hospitalization during followup. The patient was intubated 7 days after hospitalization and died after the day of intubation. Fourteenth day PCR control of 20 patients were negative, no patient had a hospitalization indication except for the patient who died.

\section{DISCUSSION}

SARS CoV-2 has deeply affected life all over the world with its rapid transmission and fatal ARDS. It is stated that the biggest factor in the transmission of the disease is asymptomatic. ${ }^{12,13}$ Although the frequency of asymptomatic infections in society is not fully known, an estimate was made based on the screening during quarantine in the Japanese cruise ship in Yokohama. The cruise ship hosting 3.711 people underwent a 2-week quarantine after a former passenger was found with COVID-19 post-disembarking. 634 people on board tested positive for the causative virus. The researchers conducted statistical modeling to derive the delayadjusted asymptomatic proportion of infections, along with the infections' timeline. The estimated asymptomatic proportion was $17.9 \% .^{14}$ And also, experts from IDSA explain that some of the asymptomatic patients were pre-symptomatic and started to spread the virus $24-48$ hours before developing symptoms.

Oncology patients have to go to the hospital due to the necessity of continuing their oncological treatments. Although the patients stated that they complied with the rules of isolation in their living spaces, we found that $15(53.5 \%)$ of 28 patients with whom they live together at home go out to work and $12(42.8 \%)$ patients came to the hospital by using public transport. So they did not fully comply with social isolation due to compulsory reasons. As asymptomatic patients are not tested, the possibility that these patients may be asymptomatic carriers or in presymptomatic period, increases the risk of infection spread to both other patients and healthcare professionals. ESMO recommends RT-PCR CoV-2 testing should be proposed to all patients undergoing surgery, radiotherapy, chemotherapy or immunotherapy, if feasible and ideally before each treatment/cycle. ${ }^{10}$

Therefore, PCR test was performed on every patient who would receive anti-cancer treatment even if they did not have symptoms suggesting COVID-19 infection. In our study, PCR test was positive in 28 of 312 patients that we tested, and the positivity rate was $8.9 \%$. Only three $(10.7 \%)$ of the oncology patients had mild symptoms, while 25 patients $(89.3 \%)$ was truly asymptomatic.

Because of the changes on computed tomography (CT) of the chest can be positive earlier than other clinical symptoms and laboratory findings, it is very useful for early diagnosis. Common abnormal radiography findings were consolidation and ground glass opacities, with bilateral, peripheral, and lower lung zone distributions. ${ }^{15}$ All PCR positive patients underwent a low-dose lung CT, and approximately half of them have COVID-19 involvement. When the limited number of literature is reviewed, a correlation is reported between the level of lung parenchymal involvement in CT and the severity of the clinical course. ${ }^{16}$ Approximately half of the PCR positive patients had no evidence of viral pneumonia. Also in those with pulmonary involvement, involvement was less than $10 \%$ of the lung. This finding may explain the low frequency of symptoms in the patient group. With the treatment given in the early period, only one of our patients required hospitalization, and the same patient died 1 week after the diagnosis.

It is not clear that, whether $89.3 \%$ of the patients had asymptomatic infection was coincidental or due to the effect of their immunosuppressive disease and treatments. Since the excessive response of the immune system is considered as one of the causes of lung damage ${ }^{17}$, impaired immune response in cancer patients may be the reason that the patients were asymptomatic. ${ }^{18}$ None of the patients developed symptoms in 14 days of followup, except for three. So these patients are in carrier position for the spread of the infection. We believe that screening tests should be performed 
at regular intervals and the isolation rules should be applied more strictly in these patients, even if they are asymptomatic before treatment, in order to continue their treatment without any problems and to prevent the risk of transmission. Also, giving chemotherapy to an asymptomatic but infected patient may increase the severity of the course of infection.

There may be some possible limitations in this study, include a single center experience and observational design without control group. It may only reflect the reality of the area and may not be a universal fact.

In summary, in oncology patients who are receiving active anti-cancer treatment without symptoms and findings suggestive of COVID-19 infection, we have to recognize the asymptomatic group of patients. For this reason, we recommend testing for COVID-19 in oncology patients actively receiving therapy, periodically or before each anticancer treatment. If we are unable to test before each treatment, it would be correct for healthcare professionals and their families to take precautions as if the patient was COVID-19 positive, in terms of the risk of contamination of the people they are in contact with.

\section{REFERENCES}

1. Surveillance case definitions for human infection with novel coronavirus (nCoV) Interim guidance v2 15 January 2020 WHO/2019-nCoV/Surveillance/v2020.2

2. World Health Organization. Director-General's remarks at the media briefing on 2019-nCoV on 11 February 2020. https:// www.who.int/dg/speeches/detail/who-director-general-sremarks-at-the-media-briefing-on-2019-ncov-on-11-february-2020 Accessed on February 12, 2020.

3. World Health Organization. Coronavirus disease (COVID-19) Pandemic. www.who.int/emergencies/diseases/novel-coronavirus-2019.

4. Perrotta F, Corbi G, Mazzeo G, et al. COVID-19 and the elderly: insights into pathogenesis and clinical decision-making. Aging Clin Exp Res 32: 1599-1608, 2020.

5. World Health Organization. Modes-of-transmission-of-viruscausing-covid-19-implications-for-ipc-precaution-recommendations. www.who.int/news-room/commentaries/detail/ modes-of-transmission-of-virus-causing-covid-19-implications-for-ipc-precaution-recommendations.
6. Onder G, Rezza G, Brusaferro S. Case-fatality rate and characteristics of patients dying in relation to COVID-19 in Italy. JAMA 323: 1775-1776, 2020.

7. Liang W, Guan W, Chen R, et al. Cancer patients in SARSCoV-2 infection: a nationwide analysis in China. Lancet Oncol 213: 335-337, 2020.

8. General Information about COVID-19 \& Cancer. https:// www.asco.org/asco-coronavirus-resources/care-individualscancer-during-covid-19/general-information-about-covid-19.

9. COVID-19 Pandemisinde Hekimlerin Almalari Gereken Kisisel Onlemler. https://www.kanser.org/saglik/haberler/covid19-pandemisinde-hekimlerin-almalari-gereken-kisisel-onlemler/2129.

10. European Society of Medical Oncology. Cancer patient management during the COVID-19 pandemic. European Society of Medical Oncology Web Site. https://www.esmo.org/ guidelines/cancer-patient-management-during-the-covid19-pandemic. 2020. Accessed April 17, 2020.

11. Infectious Diseases Society of America Guidelines on the Diagnosis of COVID-19. https://www.idsociety.org/practiceguideline/covid-19-guideline-diagnostics/ Accessed on May 08, 2020.

12. Chan JF, Yuan S, Kok KH, et al. A familial cluster of pneumonia associated with the 2019 novel coronavirus indicating person-to-person transmission: a study of a family cluster. Lancet 395: 514-23, 2020.

13. https://www.who.int/docs/default-source/coronaviruse/situation-reports/20200124-sitrep-4-2019-ncov. pdf?sfvrsn=9272d086_8

14. Mizumoto K, Kagaya K, Zarebski A, Chowell G. Estimating the asymptomatic proportion of coronavirus disease 2019 (COVID-19) cases on board the Diamond Princess cruise ship, Yokohama, Japan, 2020. Euro Surveill 25: 2000180 , 2020.

15. Shi $\mathrm{H}$, Han $\mathrm{X}$, Jiang $\mathrm{N}$, et al. Radiological findings from 81 patients with COVID-19 pneumonia in Wuhan, China: a descriptive study. Lancet Infect Dis 20: 425-434, 2020.

16. Zhao W, Zhong Z, Xie X, et al. Relation between chest CT findings and clinical conditions of coronavirus disease (COVID-19) pneumonia. AJR 214: 1072-1077, 2020

17. Xu Z, Shi L, Wang Y, et al. Pathological findings of COVID-19 associated with acute respiratory distress syndrome. Lancet Respir Med 8: 420-422, 2020.

18. Schreiber RD, Old LJ, Smyth MJ. Cancer immunoediting: Integrating immunity's roles in cancer suppression and promotion. Science 331: 1565-1570, 2011.

\section{Correspondence:}


International Journal of Hematology and Oncology

\section{Dr. Esat NAMAL}

Merkez Mahallesi, Abide-i Hürriyet Caddesi

No: 164, 34387 Sisli,

ISTANBUL / TURKEY

Tel: (+90-507) 9464716

Fax: (+90-212) 2244982

e-mail: esatnamal2000@yahoo.com

\section{ORCIDs:}

Esat Namal

Nur Dinc

Sezer Saglam

0000-0002-1954-7351

Ali Vefa Ozturk

0000-0002-1954-7351

Safiye Koculu

0000-0002-1954-7351

Asiye Yir

0000-0002-1954-7351

Esin Cevik

0000-0002-1954-7351

Soheyl Sabet

0000-0002-1954-7351

Bedriye Koyuncu Sokmen

0000-0002-1954-7351

Nagihan Inan Gurcan

0000-0002-1954-7351

Yaman Tokat

0000-0002-1954-7351

Cavlan Ciftci

0000-0002-1954-7351

0000-0002-1954-7351 